Int. J. Dev. Biol. 55: 989-993

doi: $10.1387 / \mathrm{ijdb} .113437 \mathrm{ld}$

\title{
Cloning and developmental expression of zebrafish pdzrn3
}

\author{
LUCIANA DENTE ${ }^{1}$, GAIA GESTRI ${ }^{2}$, MICHAEL TSANG3,\#, TETSUHIRO KUDOH \\ STEPHEN W. WILSON ${ }^{2}$, IGOR B. DAWID ${ }^{3}$ and MASSIMILIANO ANDREAZZOLI*,1 \\ ${ }^{1}$ Department of Biology, Universita' di Pisa, Italy, ${ }^{2}$ Department of Cell and Developmental Biology, \\ University College London, UK and ${ }^{3}$ Laboratory of Molecular Genetics, NICHD, NIH, Bethesda, MD, USA
}

\begin{abstract}
Pdzrn3, a member of the PDZRN/SEMCAP/LNX protein family containing a RING finger and two PDZ domains, has been implicated in myoblast and osteoblast differentiation. However, its spatio-temporal expression pattern during embryonic development has not been defined. Here, we describe the cloning and expression pattern of pdzrn3 during zebrafish development. We found that in addition to being expressed in several mesodermal structures, this gene displays specific expression in the central nervous system including rhombomere 1, ventral retina, thalamus and motor neurons, indicating a novel function during neural development. In particular, the absence of expression of pdzrn 3 in the ventral retina of noi mutant fish suggests a possible role for this gene in regulating fasciculation and/or navigation of retinal ganglion cell axons.
\end{abstract}

KEY WORDS: PDZRN protein, PDZ domain, RING finger, zebrafish development, ventral retina

Mechanisms involved in development and tissue differentiation are generally based on molecular recognition and formation of networks of functional interactions. The identification of genes specifically expressed in restricted regions of a tissue can help to identify interacting proteins that are determinants for given structures or functions. During the course of a large scale in situ hybridization screen for genes developmentally regulated in zebrafish embryos (Kudoh et al., 2001), we selected a gene displaying a peculiar expression in the central nervous system. Analysis of the deduced translated sequence revealed it encodes for Pdzrn3, a protein that was originally identified in silico by homology analysis to LNX1 or SEMCAP (Katoh and Katoh, 2004). PDZRN- proteins contain a RING finger and one or more PDZ domains and belong to the family of RING proteins that are generally involved in mechanisms of cell cycle progression, development, signal transduction, ubiquitination and apoptosis (Joaizero and Weissman, 2000). RING (Really Interesting New Gene) fingers are specialized structures containing a sequence motif with conserved cysteine and histidine residues related to zinc fingers (Katoh and Katoh, 2004). PDZ (PSD-95/ Discs-large/ZO-1) domains are small protein-protein interaction modules, which are often present in duplicate or in multiple copies, allowing recognition and clustering of signaling molecules for the organization of protein networks (Feng and Zhang, 2009).
Current information about Pdzrn3 mainly derives from studies in muscle tissues. Human pdzrn3 was isolated from a heart cDNA library and its expression was characterized by northern analysis in various adult human and murine tissues. It was mainly detected in heart, skeletal muscle and liver (Ko et al., 2006). The amount of pdzrn3 RNA decreases during muscle postnatal development and increases during adult muscle regeneration following injury, suggesting that the gene is developmentally regulated (Ko et al., 2006; Lu et al., 2007). Furthermore, PDZRN3 is involved in the process of differentiation of $\mathrm{C} 2 \mathrm{C} 12$ murine mesenchymal progenitor cells into myotubes or into osteoblasts (Ko et al., 2006; Honda et al., 2010). Finally, a role as an E3-ubiquitin ligase has been proposed for PDZRN3 at the level of neuromuscular junctions for the regulation of the surface expression of MuSK (Muscle-Specific receptor tyrosine Kinase; Lu et al., 2007).

Here, we describe the cloning of zebrafish pdzrn3 and its expression pattern during embryonic development. This analysis highlights a previously undescribed dynamic expression of pdzrn3 in specific domains of the developing central nervous system of zebrafish.

These data, together with the repression of $p d z r n 3$ expression in

Abbreviations used in this paper: aa, aminoacids; ac, accession number; nt, nucleotides; PDZ, PSD-95/Discs-large/ZO1; RING, really interesting new gene.

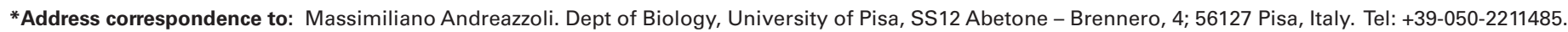
Fax: +39-050-2211495. e-mail: mandreazzoli@biologia.unipi.it

Present addresses: \#Department of Developmental Biology, University of Pittsburgh, PA, USA. \#'School of Biosciences, University of Exeter, Exeter, UK.
} 


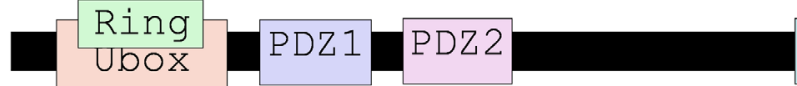

\begin{tabular}{|c|}
\hline 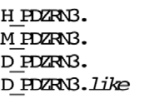 \\
\hline 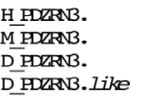 \\
\hline 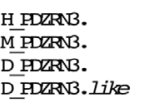 \\
\hline 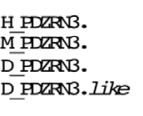 \\
\hline
\end{tabular}

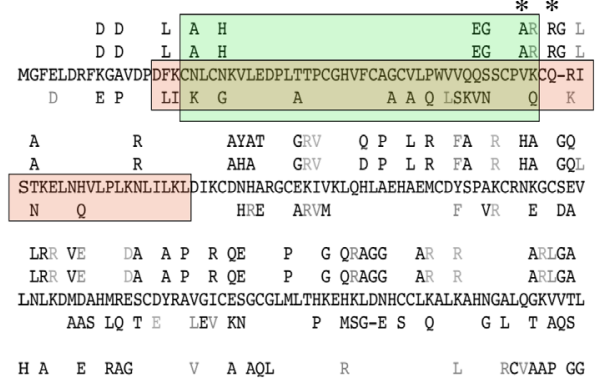

$\begin{array}{llllllll}\text { H A } & \text { E } & \text { RAG } & \text { V } & \text { A AOL } & \text { R } & \text { L } & \text { RCVAAP GG } \\ \text { H A } & \text { E } & \text { RAG } & \text { V } & \text { A AOL } & \text { R } & \text { L } & \text { RCVAAP GG }\end{array}$ DKELKKQALKSTKREKSLLAQLSAVHNELQMTALKYQKKFTEYSARIDSLSKSLSPSPPC

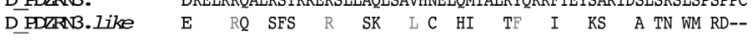

H PDZRENB.

MPDZRNS.

D FDarNB. like

H PDRRNB.

MiDRRAN3.

D PDERAN3. like

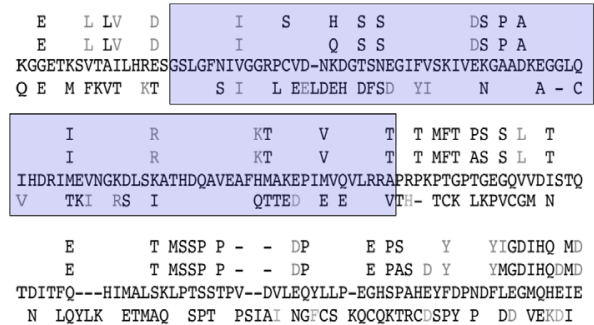

MIDERANB.

D HDRAN3.

D_PERN3.7ike

H PDEREN3.

M PDLRNB.

D IDZREN.

D_PDRFN3. like

H PDEREN.

M PDERNB.

D PDZRNB.

D HDRR 3. like

H PDERANB.

M IDZRN

D PDERN

D HDKRN3.like

H PDZRRN.

M PDERN 3 .

D PDARNB.

D PDLRN3. like

H PDERNB.

M PDZRNB

D HDZRN3. 7 ike

H HDZRN 3.

M PDERNB.

D PDER B.

DEDRR 3. like

H PDZRNB.

M PDERNB.

D PDERNB.

D EDLRN3. like

H HDZRNB.

M PDZRAN3.

D PDKRAB.

D HDERNB. like

M PDRRN

M HDKRNB.

D IDLRRN3. like

H PDRR B.

M PDERNB.

D PDERNB.

D PDKRN3. Iike

H HDRRN3.

M PDERN

D PDERN

D_ HDKRN3. like

H PDERNS.

M PDRRN 3 .

D EDLRAB.

D EDRR B. Iike

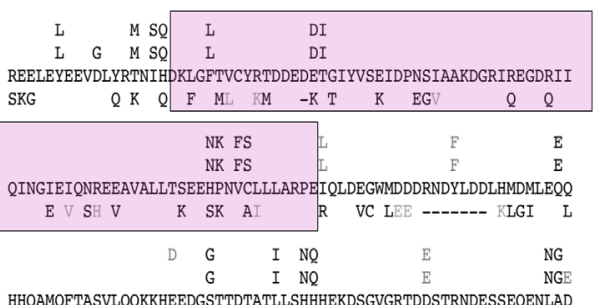

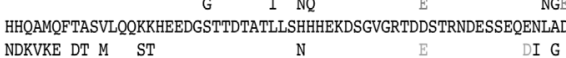

NDKVKE DT M ST

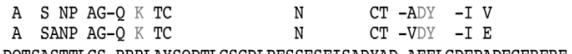

DQTSASTTLGS-RRRLAYSQDTLGSGDLPFSSESFISADYAD-AEFLGDFPADECERFRE
M E MNL N L V NG NGN I - I GTA K

VK TPY LYYP GPLDA--- KSDP $S$

$\begin{array}{ll}\text { VK } & \text { TPY LYYP GPLDA--- KSDP S } \\ V & \text { SPYSLYYP SPLDAA-- KSDP S }\end{array}$

LLELKCOMOSAGSSGTPDOSLLWPSGGOGGVGEEGVDKELELLNEELRSIELECLSIVRA

L N NNLQ LLEHDATQYGKR---ITTNCD Q DQM KQ LN IQ

$\begin{array}{llllll}K M & K & Y & \text { I V } & S & T\end{array}$

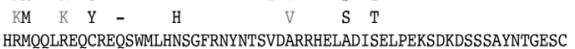

L AEDSGPQ----TTDPSTKHFIEQLNDKV QDLLNNK VDN---EC T

AA GISCPSSE VGT EAYGPAS N ITEDPEVG PTYSP VA G----SSE TANIEAYRPSP N AITEDPEVS PSYNP RSTPLTLELSPDNSLRRGNES---QAEAGASSSTSGPSRILKPLLSPVQEACSPTRGRPT W LQPA SHSPE Q MLIAD---KGKGSPMIRRNSTTACS HN IR I- S---

S LDPNQPL SK RASD S SPT SQKLGSAYLPSYHH SA LDPSOAL IK RGSD S SPTASPKLGNAYLPSYH

LLKESEVGTOPEVRERK----GGRHAHP--------------OSPYKHAHIPAHAQHYQSYM

AGDP TN GKR QS ----R RNPRS-----------LHH S NTY

A $\quad$ R LS-SPTPSE R

OLIOQKS-VEYAOSOMSLVSMCRDPV-TSADLGPKMEWKVKIRSDGTRYITKRPVRDKLL

A H V IA L KN EN TT SRL G VCN VR I $Q$

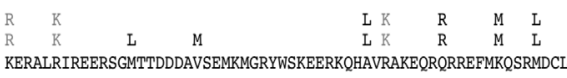

KERALRIREERSGMTTDDDAVSEMKMGRYWSKEERKOHAVRAKEORQRREFMKQSRMDCL CSI G I L N K $Q \quad$ Q $Q$ LI CY ENK

Q-QAADDR EM

Q-QASDDR EM

KELGVTTEAKKDVNIIELSHKKMMKKRNKKIFDNWMTIOELLTHGTKSPDGTRVYNSLLS

GKT -- LNN EPD $\quad 0 \quad R$

* PDZ-binding

VTTV* motif the ventral eye region of zebrafish mutants affecting eye development, suggest that this gene might be involved in controlling neuritogenesis and in particular axon outgrowth of retinal ganglion cells.

\section{Results}

\section{Cloning of zebrafish pdzrn3}

The pdzrn3 gene was selected for its peculiar expression in the developing eye of zebrafish in the course of a large scale in situ hybridization screen (Kudoh et al., 2001). A complete coding clone of 3162 nucleotides (nt), was identified by 5'RACE and end-to-end PCR (GenBank accession no. JN108761). Sequence analysis revealed an open reading frame encoding a predicted protein of 1053 aminoacids (aa) (Fig. 1). A database search provided precise alignment with a predicted zebrafish pdzrn3 sequence (GenBank accession no. XM_001344515), derived from assembled genomic sequences and supported by EST evidence. The two sequences share $99 \%$ identity, diverging in 14 nucleotides that determine 6

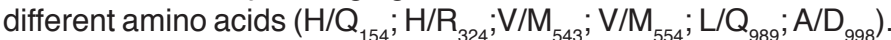
The pdzrn3 gene is localized on zebrafish chromosome 6 (http:// www.ncbi.nlm.nih.gov/gene/100005518). The zebrafish genome often displays more alleles of genes that are present in single copy in mammals. In this case, a related gene, named pdzrn3like has been reported on chromosome 23 (http://www.ncbi.nlm. nih.gov/gene/100149029). The corresponding protein Pdzrn3-like (XP_001921128.2), deduced from translated genomic and EST sequences, diverges from our Pdzrn3 sequence in multiple positions sharing only $54 \%$ identity. Comparison with human and murine PDZRN3 proteins revealed higher homology with zebrafish Pdzrn3 protein (72\%) than with Pdzrn3-like (50\%) (Fig. 1). Homology with murine PDZRN4 (XP_997312) is even lower: 51\% for Pdzrn3 and $37 \%$ for Pdzrn3-like. Only in the zebrafish proteins a potential U box overlapping the Ring finger motif is present. The residues $\mathrm{V}_{54}$ and $Q_{57}$ are determinants for $U$ box prediction according to SMART (http://smart.embl-heidelberg.de/). $U$ boxes are domains deriving from RING fingers, which miss the full complement of $\mathrm{Zn}^{2+}$-binding ligands. It has been proposed that $U$ box scaffold has evolved from a RING finger domain by appropriation of a new set of residues required to stabilise its structure through a system of salt-bridges and hydrogen bonds (Aravind and Koonin, 2000). U-box proteins have been reported to function as ubiquitin-protein ligases (E3s) and to interact with molecular chaperones. The presence of both RING and U-box domains strengthens the hypothesis that Pdzrn3 is an ubiquitin ligase involved in the degradation of unfolded or misfolded proteins.

\section{Developmental expression pattern of pdzrn3}

The pdzrn3 spatio-temporal expression pattern was analyzed

Fig. 1. Sequence and structure of zebrafish Pdzrn3. Predicted domain composition of Pdzrn3 (top) and comparison of amino acid sequences: H_PDZRN3 (human, ac.Q9UPQ7); M_PDZRN3 (murine, ac.Q69ZS0): D_PDZRN3 (zebrafish, translated from ac. JN108761) and D_PDZRN3. like (predicted by automated computational analysis, ac.XP_001921128.2). The amino acid sequence deduced from the pdzrn3 cDNA we have cloned is completely shown. Similar amino acids of the aligned proteins are indicated in gray, different aminoacids are in black, whereas identical residues of the listed proteins are shown only in the zebrafish Pdzrn3 sequence. Asterisks indicate the critical residues involved in the U-box domain structure. Predicted domains deriving by SMART analysis (http:// smart.embl-heidelberg.de) are boxed. 
using wholemount in situhybridization. pdzrn3transcripts are initially detected at the 3-somite stage in two areas of the presumptive hindbrain and in the notochord (Fig. 2A). By 6-somite stage the two hindbrain sites of expression fuse medially and at 10-somite stage pdzrn3 begins to be expressed in the ventral region of the optic primordia (Fig. 2B and data not shown). These expression sites are maintained in the following stages (Fig. 2C-E). Prolonged staining at 20-somite stage reveals expression also in somites and in posterior notochord (Fig. 2F). At 24 hpf, pdzrn3 displays a clear expression in the ventral retina and optic stalk, and, at a lower level, in the dorsal region of the retina (Fig. $2 \mathrm{H}$ ). Acomparison with pax2.1a expression at $24 \mathrm{hpf}$ highlights the absence of pdzrn3 expression in more medial regions of the optic stalk, which are labeled by pax2.1a(Fig. $2 \mathrm{G}, \mathrm{H}$ ). Double in situhybridizations with pax2.1aand krox20 (also known as egr2b), markers of the midbrain-hindbrain boundary and of rhombomere 3 and 5, respectively, show that the hindbrain expression domain of $p d z r n 3$ corresponds to the lateral regions of rhombomere 1 (Fig. 2 I,J). At 24 hpf, prolonged staining reveals expression in ventral spinal cord neurons and in cells of the ventral somites (Fig. 2K). By 32hpf pdzrn3 expression in the eye lines the choroid fissure, and now also shows expression in ganglion cells. At this stage, pdzrn3 begins to be expressed
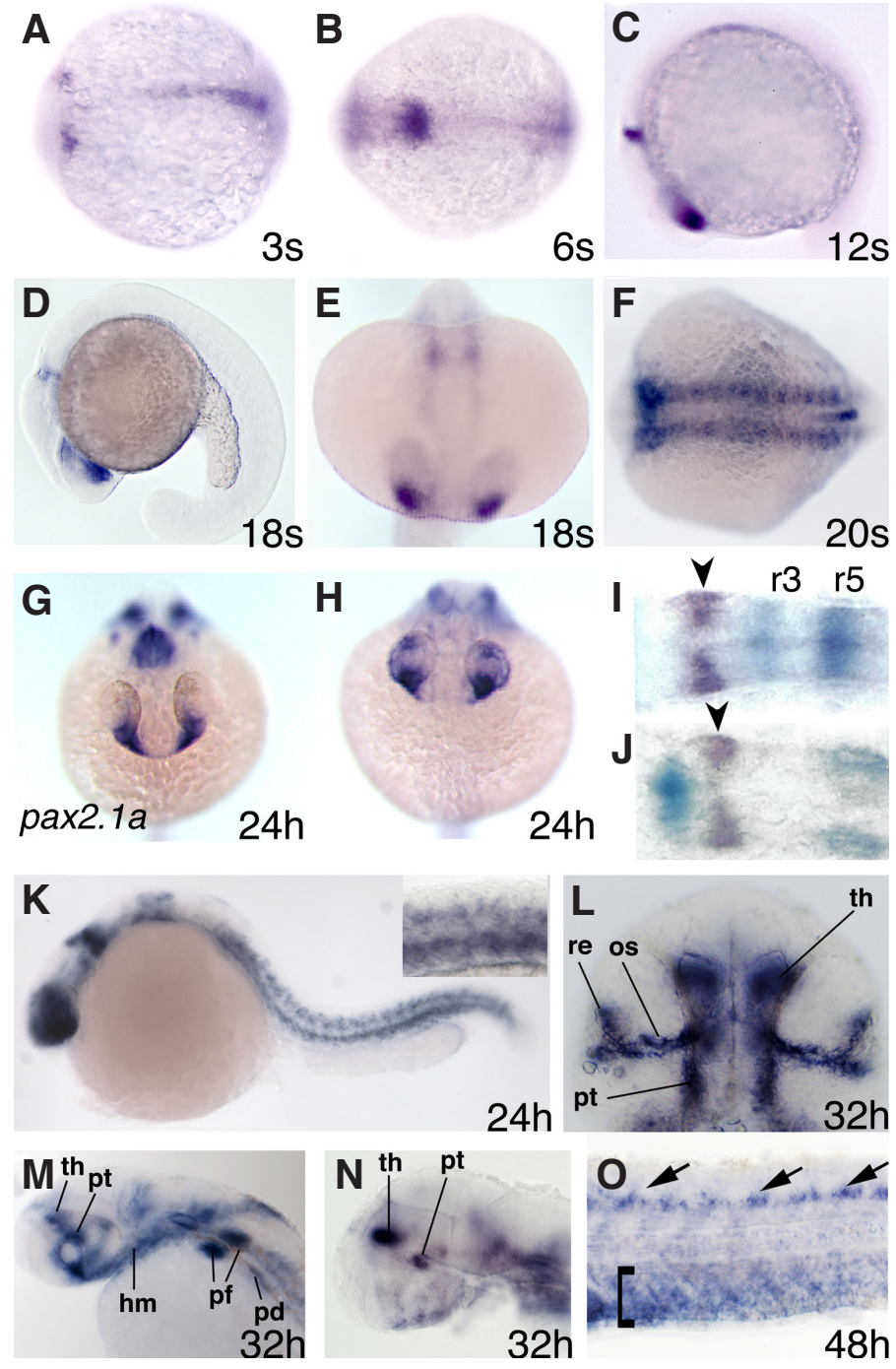

also in the thalamus and in the posterior tuberculum, as well as in head mesenchyme, pectoral fins and pronephric duct (Fig. 2 $\mathrm{L}-\mathrm{N})$. The same expression sites are present at $48 \mathrm{hpf}$ when the neural expression in trunk-tail becomes more clearly restricted to motor neurons (Fig. 20 and data not shown).

\section{Analysis in zebrafish mutants}

As a preliminary analysis to identify factors regulating pdzrn3 expression in the central nervous system, we took advantage of zebrafish mutants affecting different genes involved in retina and brain development. pdzrn3 expression does not appear to be particularly affected in you-too (yot), acerebelar(ace), chameleon(con), and cyclops (cyc) mutants, and only slightly affected in smoothened (smo) mutants (Fig. 3 C,D and data not shown; Hatta et al., 1994; Brand et al., 1996; Barresi et al., 2000). On the contrary, a more specific effect is observed in no isthmus (nol) mutants that show a regionally restricted lack of pdzrn3 expression in the ventral retina and distal optic stalk (Fig. $3 \mathrm{G}, \mathrm{H}$ ). Notably, in noi mutants optic axons display fasciculation and pathfinding defects in these positions (Macdonald et al., 1997).

\section{Discussion}

In this work, we describe the cloning of pdzrn3 and show that this gene displays a dynamic expression during zebrafish development. Although some studies have addressed the function of this gene, in particular in the context of murine muscle differentiation/regeneration and osteogenesis, its spatio-temporal expression pattern during embryogenesis was not previously described. We found that $p d z r n 3$ is expressed in several mesodermal and neuroectodermal sites. By in situ hybridization, the earliest detected signal in somites appears at the 20-somite stage, much later than the onset of expression of the muscle determinants myoD and myogenin (Weinberg et al., 1996). This is in keeping with functional studies of mouse Pdzrn3 by Ko et al., (2006), indicating that this gene may act downstream or independently of $M y O D$ and Myogenin. An early mesodermal site of expression is the developing notochord. As development proceeds, pdzrn3 expression is progressively restricted to more posterior regions of the notochord. Further mesodermal expression sites become evident only at $24 \mathrm{hpf}$ and include cells in the ventral

Fig. 2. Expression pattern of pdzrn3. $(\mathbf{A}, \mathbf{B})$ Three-somite $(A)$ and sixsomite (B) stage: dorsal views, anterior to the left. (C-E) twelve-somite (C) and eighteen-somite (D) stage: lateral views, anterior to the left. (E) Eighteen-somite stage: dorsal view, anterior to the bottom. (F) Twentysomite stage embryo stained for prolonged time: dorsal view, anterior to the left. $(\mathbf{G}, \mathbf{H})$ Comparison between the anterior expression of pax $2.1 \mathrm{a}$ (G) and pdzrn3 (H) at 24-hpf stage: frontal views, dorsal to the top. $(\mathbf{I}, \mathbf{J})$ Comparison between the rhomboencephalic expressions of pdzrn3 (I, J; purple staining indicated by arrowheads), krox20 (I, turquoise staining) and pax2.1a (J, turquoise staining); dorsal view, anterior to the right. (K) 24-hpf stage embryo stained for prolonged time: lateral view, anterior to the left. The inset in the top right corner shows a magnification of somite expression in the trunk. (L) Head expression in 32 hpf embryo: ventral view anterior to the top; os, optic stalk; pt, posterior tuberculum; re, retina; th, thalamus. (M,N) 32 hpf stage: lateral view, anterior to the left; hm, head mesenchyme; $p d$, pronephric duct; pf, pectoral fin. To analyze internal brain expression sites the eyes of the embryo shown in $N$ were removed. (0) Trunk and tail expression at 48 hpf: dorsal to the top, anterior to the left. Arrows point to the motor neurons of the spinal cord. The bracket delimits pdzrn3 expression in the ventral region of somites. 
A

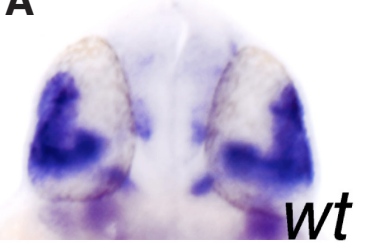

C

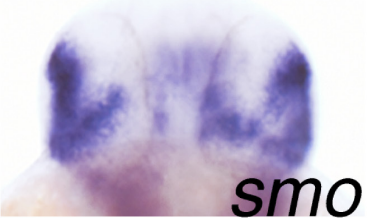

E
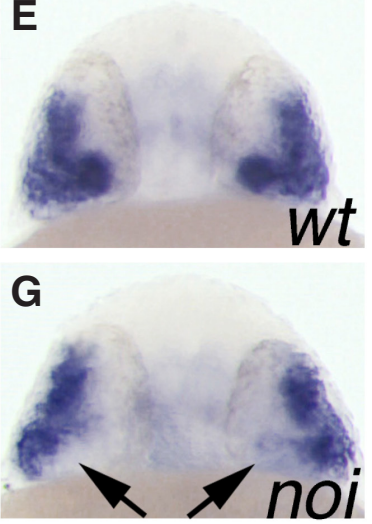

B

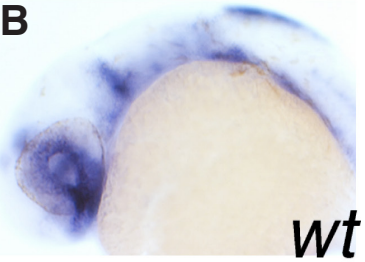

D
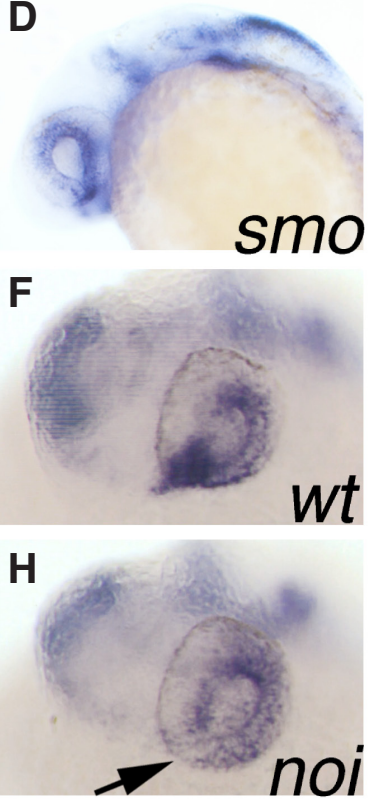

Fig. 3. Retinal expression of pdzrn3 in selected zebrafish mutants. (A-H) Retinal expression of pdzrn3 at 24-hpf stage in wild type (A,B,E,F), smo $(\mathbf{C}, \mathbf{D})$ and noi mutants $(\mathbf{G}, \mathbf{H})$. $(A, C, E, G)$ ventral view, anterior to the top. $(B, D)$ Lateral view, anterior to the left $(F, H)$ latero-anterior view, dorsal to the top. Arrows in $(G, H)$ point to the lack of pdzrn3 expression in the ventral retina of noi mutants.

somites, head mesenchyme and pronephric duct.

A previously undescribed feature of $p d z r n 3$ expression, highlighted by this study, is its expression in the central nervous system. In fact, pdzrn3 is expressed in presumptive rhombomere 1 starting from 3-somite stage and in the ventral optic primordia starting from 10-somite stage and, later on, in thalamus and motor neurons. Because of the early expression in the presumptive ventral retina, pdzrn3 is potentially involved in the initial dorso-ventral patterning of this organ. As pdzrn3 was shown to counteract the effect of BMP2 in osteogenesis, it is tempting to speculate that it may play the same role in the retina antagonizing the BMP signal, a key determinant of retina dorso-ventral polarity (French et al., 2009; Honda 2010). At $24 \mathrm{hpf}, p d z r n 3$ is expressed in the ventral retina and at the optic nerve head potentially including the first ganglion cells that are generated at this stage in the ventral retina (Stenkamp, 2007). At $32 \mathrm{hpf}, p d z r n 3$ expression is extended to retinal ganglion cells as well as cells lining the choroid fissure and to the thalamus. Notably, among the analyzed mutants, the noi mutant displays the most specific repression of pdzrn3 expression. This may indicate that pdzrn3 acts downstream of pax2.1a (the gene mutated in noi), which encodes a transcription factor controlling ventral retina morphogenesis and choroid fissure closure, optic stalk differentiation and axon fasciculation (Macdonald etal., 1997).

A role for pdzrn3 in axon development is possible as Semcap1, another member of the pdzrn/LNX protein family was shown to interact with Semaphorin 4C (Wang et al., 1999).

\section{Materials and Methods}

\section{Vectors and clones}

A partial pdzrn3 cDNA clone was initially isolated from a 10-somite zebrafish cDNA library by randomly picking isolated colonies in the course of an in situ hybridization screening (Kudoh et al., 2001). 5' RACE PCR was performed using the SMART RACE cDNAAMPLIFICATION KIT (Clontech) and the following pdzrn3 specific primer: 8RACE-1 GTCACCACTGCCAAGGGTGTCCTG. A complete coding pdzrn3 cDNA was obtained by end-to-end PCR performed with primers EE-FATAATGGGATTTGAGTTGGATCGTTTC and EE-RGAGCCTACACTGTAGTCACAGAGAGTAGGGA The resulting CDNA was cloned in pGEM-T easy vector (Promega).

\section{Zebrafish embryo manipulation and in situ hybridization}

Embryo generation, staging and in situ hybridization were performed as described in Andreazzoli et al., (2001). BM purple and BCIP (Roche) were used as alkaline phosphatase substrates.

\section{Acknowledgements}

We thank Giuseppina Barsacchi for helpful suggestions and Elena Landi, Marzia Fabbri and Donatella De Matienzo for technical assistance. We also gratefully acknowledge Maria Elena Terreni for her help with some in situ hybridization experiments.

This work was supported by the Intramural Research Program of the National Institute of Child Health and Human Development, NIH, by a grant from MIUR to M.A. and by grants from Telethon and MRC to G.G.

\section{References}

ANDREAZZOLI, M., BROCCOLI, V., DAWID, I.B. (2001). Cloning and expression of noz1, a zebrafish zinc finger gene related to Drosophila nocA. Mech Dev 104: 117-120.

ARAVIND, L., KOONIN, E.V. (2000). The U box is a modified RING finger - a common domain in ubiquitination. Curr Biol 10: R132-134.

BARRESI M.J., STICKNEY H.L., DEVOTO S.H. (2000). The zebrafish slow-muscleomitted gene product is required for Hedgehog signal transduction and the development of slow muscle identity. Development 127: 2189-2199.

BRAND M., HEISENBERG C.P., WARGA R.M., PELEGRI F., KARLSTROM R.O., BEUCHLE D., PICKER A., JIANG Y.J., FURUTANI-SEIKI M., VAN EEDEN F.J., GRANATO M., HAFFTER P., HAMMERSCHMIDT M., KANE D.A., KELSH R.N., MULLINS M.C., ODENTHAL J., NÜSSLEIN-VOLHARD C. (1996). Mutations affecting development of the midline and general body shape during zebrafish embryogenesis. Development 123: 129-142.

FENG W., ZHANG M. (2009). Organization and dynamics of PDZ-domain-related supramodules in the postsynaptic density. Nat Rev Neurosci 10: 87-99.

FRENCH C.R., ERICKSON T., FRENCH D.V., PILGRIM D.B., WASKIEWICZ A.J. (2009). Gdf6a is required for the initiation of dorsal-ventral retinal patterning and lens development. Dev Biol 333: 37-47.

HATTA K., PÜSCHEL A.W., KIMMEL C.B. (1994). Midline signaling in the primordium of the zebrafish anterior central nervous system. Proc Natl Acad Sci USA 91: 2061-2065.

HONDA T., YAMAMOTO H., ISHII A., INUI M. (2010). PDZRN3 negatively regulates BMP-2-induced osteoblast differentiation through inhibition of Wnt signaling. Mol Biol Cell 21: 3269-3277.

JOAZEIRO C.A., WEISSMANA.M. (2000). RING finger proteins: mediators of ubiquitin ligase activity. Cell 102: 549-552.

KATOH M., KATOH M. (2004). Identification and characterization of PDZRN3 and PDZRN4 genes in silico. Int J Mol Med 13: 607-613.

KO J.A., KIMURA Y., MATSUURA K., YAMAMOTO H., GONDO T., INUI M. (2006). PDZRN3 (LNX3, SEMCAP3) is required for the differentiation of $\mathrm{C} 2 \mathrm{C} 12$ myoblasts into myotubes. J Cell Sci 119: 5106-5113. 
KUDOH T., TSANG M., HUKRIEDE N.A., CHEN X., DEDEKIAN M., CLARKE C.J., KIANG A., SCHULTZ S., EPSTEIN J.A., TOYAMA R., DAWID I.B. (2001) A gene expression screen in zebrafish embryogenesis. Genome Res. 11: 1979-1987.

LU Z., JE H.S., YOUNG P., GROSS J., LU B., FENG G. (2007). Regulation of synaptic growth and maturation by a synapse-associated $\mathrm{E} 3$ ubiquitin ligase at the neuromuscular junction of protein kinase D. J Biol Chem 177: 1077-1089.

MACDONALD R., SCHOLES J., STRÄHLE U., BRENNAN C., HOLDER N., BRAND M., WILSON S.W. (1997). The Pax protein Noi is required for commissural axon pathway formation in the rostral forebrain. Development 124: 2397-2408.
STENKAMP D.L. (2007). Neurogenesis in the fish retina. Int Rev Cytol 259: 173-224 WANG L.H., KALB R.G., STRITTMATTER S.M. (1999). A PDZ protein regulates the distribution of the transmembrane semaphorin, M-SemF J Biol Chem 274: 14137-14146.

WEINBERG E.S., ALLENDE M.L., KELLY C.S., ABDELHAMID A., MURAKAMI T., ANDERMANN P., DOERRE O.G., GRUNWALD D.J., RIGGLEMAN B. (1996). Developmental regulation of zebrafish MyoD in wild-type, no tail and spadetai embryos. Development 122: 271-280.

Further Related Reading, published previously in the Int. J. Dev. Biol.

Cytoskeletal heart-enriched actin-associated protein (CHAP) is expressed in striated and smooth muscle cells in chick and mouse during embryonic and adult stages

Willemijn van Eldik, Abdelaziz Beqqali, Jantine Monshouwer-Kloots, Christine Mummery and Robert Passier

Int. J. Dev. Biol. (2011) 55: 649-655

Expression of protocadherin-19 in the nervous system of the embryonic zebrafish Qin Liu, Yun Chen, Fumitaka Kubota, Jean J. Pan and Tohru Murakami Int. J. Dev. Biol. (2010) 54: 905-911

Expression of protocadherin 18 in the CNS and pharyngeal arches of zebrafish embryos Fumitaka Kubota, Tohru Murakami, Yuki Tajika and Hiroshi Yorifuji Int. J. Dev. Biol. (2008) 52: 397-405

Retinoic acid is required for specification of the ventral eye field and for Rathke's pouch in the avian embryo Malcolm Maden, Aida Blentic, Susan Reijntjes, Sophie Seguin, Emily Gale and Anthony Graham Int. J. Dev. Biol. (2007) 51: 191-200

Cloning, expression and functional study of translation elongation factor 2 (EF-2) in zebrafish Shu-hong Zhang, Ji-hua Yao, Huai-dong Song, Lu Wang and Jing-lun Xue Int. J. Dev. Biol. (2006) 50: 399-403

5 yr ISI Impact Factor $(2010)=2.961$

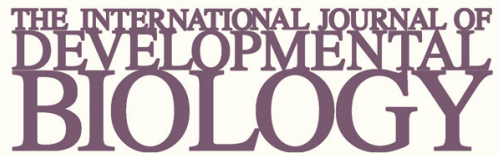

Volume 54 Nos. 6/7

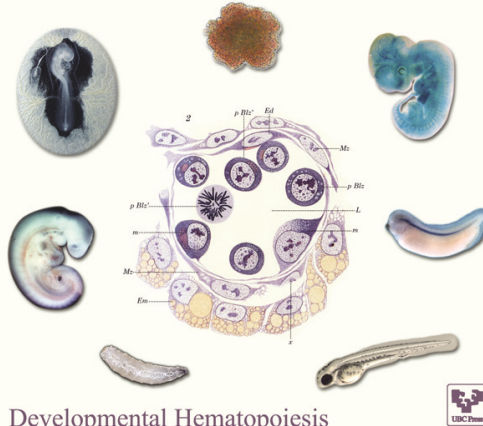

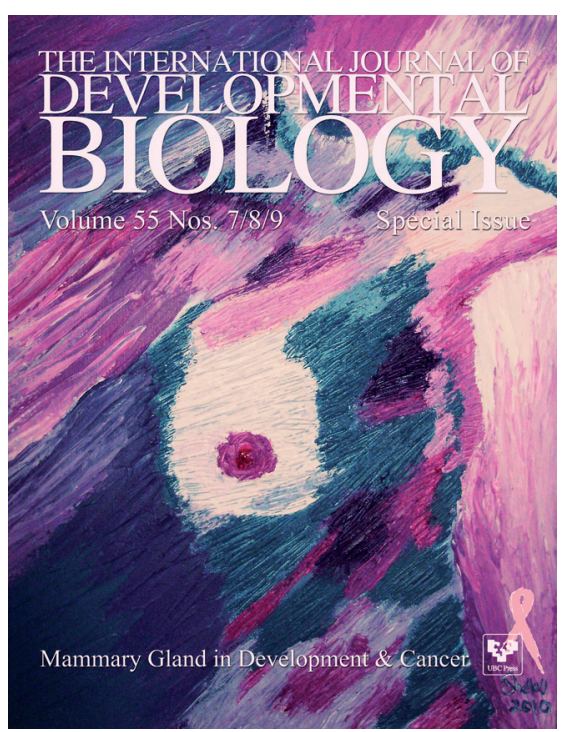

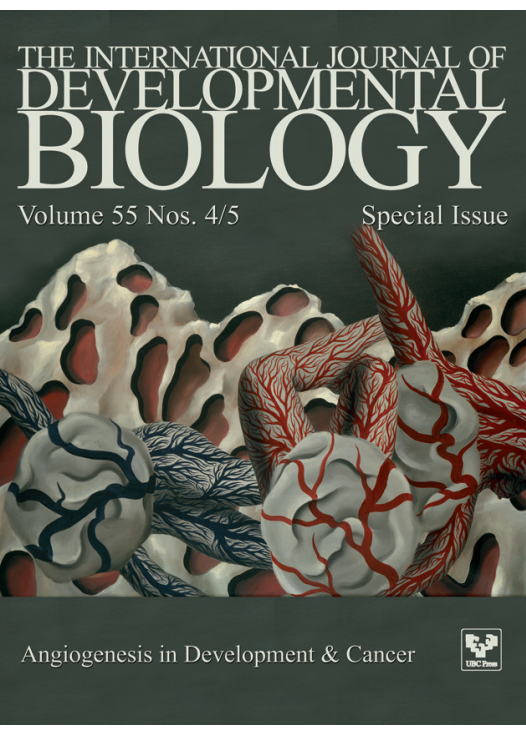

